\title{
Simulations of Re-Entry Vehicles by Using DSMC with Chemical-Reaction Module
}

\author{
Kun-Chang Tseng* \\ National Space Organization, Hsinchu 30078, Taiwan \\ Jong-Shinn $\mathrm{Wu}^{\dagger}$ \\ National Chiao-Tung University, Hsinchu 30050, Taiwan \\ and \\ Iain D. Boyd \\ University of Michigan, Ann Arbor, Michigan, 48109, USA
}

\begin{abstract}
In some applications of rarefied gas dynamics, including flows in aerospace/space engineering, film deposition technique in semiconductor and micro-reactor in medical science, chemical reactions play an important role and can not be ignored in these simulations. To understand effects of chemical reactions in these fields by experiment is very expensive and impractical. Thus, an appropriate simulation method is worthy to develop. A particle method named the direct simulation of Monte Carlo method (DSMC) is used to simulate these complicated flows and chemical-reaction module can be incorporated properly in the molecular level. In the current study, a chemical-reaction module, including dissociation, exchange and recombination for air species, is developed and implemented into a previously developed general-purpose parallel DSMC code (PDSC). The total collision energy model (TCE) is used to calculate the reaction probabilities for dissociation and exchange reactions. A three-body collision model, proposed by Boyd, is utilized to treat the recombination reaction. The reaction probability of each chemical reaction and the degree of dissociation of a single-cell are used to verify the chemical-reaction module by comparing with theoretical data. Then three different types of hypersonic re-entry vehicles, including sphere, blunt-body and Apollo, are simulated to understand the effects of chemical reactions
\end{abstract}

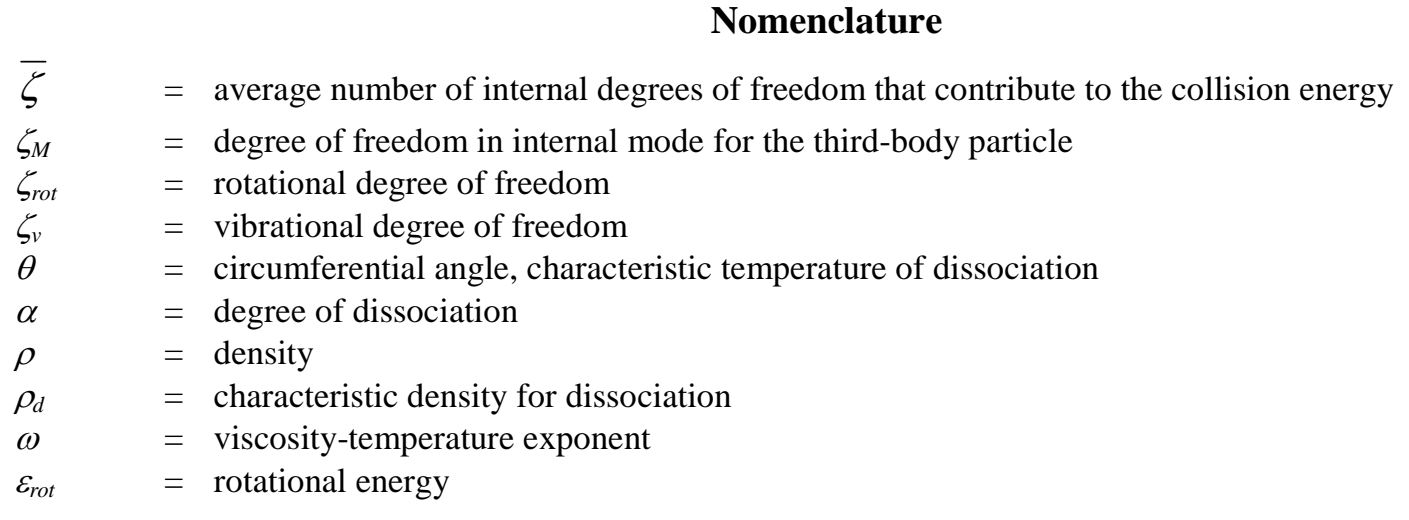

\footnotetext{
* Assistant Researcher, Department of Mechanical Engineering, 8F, 9 Prosperity 1st Road, Hsinchu Science Park, Hsinchu 30078, Taiwan

${ }^{\dagger}$ Professor, Department of Mechanical Engineering, National Chiao-Tung University, 1001 Ta-Hsueh Road, Hsinchu 30050, Taiwan

* Professor, Department of Aerospace Engineering, University of Michigan, Ann Arbor, FXB Building 1320 Beal Avenue Ann Arbor, Michigan 48109-2140, USA
} 


\begin{tabular}{|c|c|c|}
\hline$\varepsilon_{v}$ & $=$ & vibrational energy \\
\hline$\sigma_{r e f}$ & $=$ & the reference cross section for atom-atom collision \\
\hline$\sigma_{T}$ & $=$ & the total cross section \\
\hline$\Delta t$ & $=$ & time-step \\
\hline$a_{c}$ & $=$ & constant for equilibrium rate reaction \\
\hline$a_{f}$ & $=$ & constant for forward rate reaction \\
\hline$b_{c}$ & $=$ & constant for equilibrium rate reaction \\
\hline$b_{f}$ & $=$ & constant for forward rate reaction \\
\hline$c_{r}$ & $=$ & relative velocity \\
\hline$d_{r e f}$ & $=$ & reference diameter \\
\hline$E_{a}$ & $=$ & activation energy \\
\hline$E_{c}$ & $=$ & total collision energy \\
\hline$k$ & $=$ & Boltzmann constant \\
\hline$k_{c}$ & $=$ & equilibrium rate constant \\
\hline$k_{f}$ & $=$ & forward rate constant \\
\hline$k_{b}$ & $=$ & backward rate constant \\
\hline$K n$ & $=$ & Knudsen number \\
\hline$m$ & $=$ & molecular mass \\
\hline$m_{r}$ & $=$ & reduce mass \\
\hline$n$ & $=$ & number density \\
\hline$n_{N}$ & $=$ & number density of atomic nitrogen \\
\hline$n_{M}$ & $=$ & number density of the third body \\
\hline$N$ & $=$ & simulated particle number \\
\hline$N^{a}$ & $=$ & particle number of dissociated A-atoms \\
\hline$N_{A}$ & $=$ & total number of $\mathrm{A}$-atoms present in the mixture \\
\hline$P_{r}$ & $=$ & reaction probability \\
\hline $\operatorname{Re}$ & $=$ & Reynolds number \\
\hline$T$ & $=$ & temperature \\
\hline$T_{r e f}$ & $=$ & reference temperature for atom-atom collision \\
\hline$T_{\text {rot }}$ & $=$ & rotational temperature \\
\hline$T_{t o t}$ & $=$ & overall temperature \\
\hline$T_{t r}$ & $=$ & translational temperature \\
\hline$T_{v}$ & $=$ & vibrational temperature \\
\hline
\end{tabular}

\section{Introduction}

$\mathrm{T}$

HE direct simulation Monte Carlo (DSMC) method [1,2] is widely used in simulating rarefied gas flows. The examples include the pumping characteristics of turbo-molecular drag vacuum pump [3], rarefied hypersonic flow [4] and the micro-electro-mechanical-system (MEMS) [5], etc.. Most applications are simulated by the DSMC method without chemical reactions because those are non-reactive flows. But chemical reactions may occur when the flow velocity and temperature become very high. In some applications of rarefied gas dynamics, including flows in aerospace/space engineering, film deposition technique in semiconductor and micro-reactor in medical science [6-8], chemical reactions have to be considered in these applications. Fortunately, chemical reactions can be properly included in the DSMC for those complicated flows.

The total collision energy model (TCE) is proposed by Bird [1,2], which indicates the reaction probability is a function of total collision energy and coefficients of Arrhnius equation. Dogra et al. used this chemical model to simulate a 1.6-meter-diameter sphere in hypersonic rarefied flow [6]. Boyd also developed a chemistry model, which is so called vibrationally-favored dissociation (VFD). A three-body recombination reaction is also present in this paper [9]. A new modified reaction model is also proposed to figure out systematic errors when used with discrete energy modes by Ivanov's group [10].

In the current paper, we focus on developing and verifying the chemical-reaction module. Then several hypersonic re-entry flows, which the temperature is high enough to activate chemical reactions, are studied. This is very important because it is concerned with the safety of boarding astronauts. The organization of this paper is shown as follow; first is the numerical method, which includes introductions of the parallel DSMC code (PDSC) and 
chemical-reaction module. Then the code will be verified and three applications are presented in turn. Finally, conclusions are made.

\section{Numerical Method}

The direct simulation Monte Carlo (DSMC) method, which is a powerful particle method, is proposed by Bird in 1976 to simulate rarefied gas flows [1]. Although it does not solve the Boltzmann equation directly, Nanbu and Wanger $[11,12]$ both proved DSMC does provide solutions that are consistent with the Boltzmann equation. A general-purpose parallel DSMC code (PDSC), which is proposed by Wu's group [13-15], is used in this study and has several important features as follows; (a) using unstructured tetrahedral or hexahedral mesh for better treatment of complex geometry of boundaries; (b) using pressure boundary treatment in dealing flow with input/output pressure boundaries; (c) parallel computing with dynamic domain decomposition for a fast and load-balancing simulation; (d) using variable time-step scheme, in combination with adaptive mesh refinement, to reduce the simulation particles and to increase the accuracy of the numerical solution; (e) using conservative weighting scheme to efficiently treat gas flows with trace species and (f) combining the chemical reactions for simulating hypersonic air flows. This code has been used to simulate several applications to show its efficient and accurate capabilities [1315].

Chemical reactions in PDSC, involving dissociation, exchange and recombination is collaborated with Professor Boyd at the University of Michigan. For the dissociation and exchange reactions, we used the total collision energy (TCE) model to model the reaction probability [1-2]. The only difference between these two reactions is the constants of the Arrhenius equation. A tree-body collision model is proposed by Boyd to simulate the recombination, which is an extended model of the TCE model [9]. In this method, the two reactant atoms process binary collision first, which the TCE model is valid. Then a third collision body is chosen at random in the current cell to complete the ternary collision. The details can be found in a relevant paper [9]. The flow chart of chemistry in PDSC is shown in Fig. 1 and the process is described sequentially as follows: (a) Selecting two particles randomly in the current cell and determining the type of chemical reaction by the selected collision pairs. (b) Calculating the total energy and compare with activation energy of the specific chemical reaction. Calculating the reaction probability that fellows if the total energy is larger than the activation energy. If not, the normal elastic collision and energy redistribution are processed as usual. (c) Using the Acceptant-Rejection method [1-2] to determine whether the chemistry occurs or not. If the chemical reaction occurs, assign energies, velocities and positions to product particles. Or, elastic collision and energy redistribution are processed. (d) Processing the next collision until all the collision pairs are handled for all cells. The detailed derivations and validations are shown in the following section.

\section{A. Dissociation reaction}

A typical bimolecular reaction can be written as

$$
A+B \leftrightarrow C+D
$$

where A, B, C, D represent separate molecular species, which can be molecules, atoms, ions and photons. The rate equation for the change in number density of species A can be written

$$
-\frac{d n_{A}}{d t}=k_{f}(T) n_{A} n_{B}-k_{b}(T) n_{C} n_{D}
$$

$k_{f}$ and $k_{b}$ are the forward and reverse rate constants as a function of temperature, which can be expressed in the following form (Arrhenius equation)

$$
k_{f}=a T^{b} \exp \left(-\frac{E_{a}}{k T}\right)=a T^{b} \exp \left(-\frac{\theta}{T}\right)
$$

where $a, b$ are constants, and $\theta \equiv E_{a} / k$ is the characteristic dissociation temperature. $E_{a}$ and $k$ are the activation energy and Boltzmann constant, respectively. These values are usually obtained from experimental data.

From classical collision theory, the forward reaction may be written [16]

$$
k_{f} n_{A} n_{B}=n_{A} v_{A B} \int_{\frac{E_{c}}{k T}}^{\infty} f\left(\frac{E_{c}}{k T}\right) P_{r}\left(\frac{E_{c}}{k T}\right) d\left(\frac{E_{c}}{k T}\right)
$$

$v_{A B}$ is the collision rate between one A particle with all B particles in the volume, which may be expressed as 


$$
v_{A B}=\frac{n_{B} \overline{\sigma_{T} c_{r}}}{\alpha} \quad \alpha=1 \text { for } A \neq B, \quad \alpha=2 \text { for } A=B
$$

$n_{B}$ and $\alpha$ are the number density of $\mathrm{B}$ particles and the symmetric factor, respectively. $\sigma_{T}$ is the total collision cross section and $c_{r}$ is the relative velocity of collision pair. Thus, $\overline{\sigma_{T}} c_{r}$ is the average volume that one A-B particle pair sweeps per unit time, which can be written as

$$
\overline{\sigma_{T} c_{r}}=\frac{2 \sigma_{r e f}}{\sqrt{\pi}}\left[(2-\omega) T_{r e f}\right]^{\gamma} \Gamma(2-\gamma)\left(\frac{2 k}{m_{r}}\right) T^{1 / 2-\gamma}
$$

$\sigma_{r e f}, T_{r e f}$ and $m_{r}$ are the reference cross section, temperature and reduce mass, respectively. By substitution of Eqs. (5) and (6) into Eq. (4), the dissociation probability (steric factor) can be obtained

$$
\begin{gathered}
k_{f} n_{A} n_{B}=n_{A} v_{A B} \int_{\frac{E_{c}}{k T}}^{\infty} f\left(\frac{E_{c}}{k T}\right) P_{r}\left(\frac{E_{c}}{k T}\right) d\left(\frac{E_{c}}{k T}\right)=n_{A} \frac{n_{B} \overline{\sigma_{T} c_{r}}}{\alpha} \int_{\frac{E_{c}}{k T}}^{\infty} f\left(\frac{E_{c}}{k T}\right) P_{r}\left(\frac{E_{c}}{k T}\right) d\left(\frac{E_{c}}{k T}\right) \\
P_{r}\left(\frac{E_{c}}{k T}\right)=\frac{\alpha a \sqrt{\pi}}{2 \sigma_{r e f}} \frac{\Gamma(\bar{\zeta}+2-\gamma)}{\Gamma(2-\gamma) \Gamma\left(\bar{\zeta}+\frac{3}{2}+b\right)}\left(\frac{m_{r}}{2 k}\right)^{1 / 2-\gamma} \times \\
{\left[\frac{m_{r}}{2(2-\omega) k T_{r e f}}\right]^{\gamma}\left(1-\frac{E_{a}}{E_{c}}\right)^{\bar{\zeta}+1-\gamma}\left(\frac{E_{c}-E_{a}}{k}\right)^{b-1 / 2+\gamma}}
\end{gathered}
$$

where $\bar{\zeta}=\left(\zeta_{\mathrm{A}}+\zeta_{\mathrm{B}}\right) / 2$ is the average number of internal degree of freedom that contribute to the collision energy. $\omega=\gamma+1 / 2$ is the viscosity-temperature exponent, which value can be found in the Appendix of the Bird's book. Equation 7 is the so called the total collision energy (TCE) model [2]. Thus, Eq. (7) is the dissociation probability that we are used for the current DSMC code.

In the DSMC simulation, the reaction probability is obtained by accumulating the number of collisions between reactants and the number of collisions that do react. Then the rate constant can be calculated by using Eq. (8)

$$
k_{f}=\frac{\overline{\sigma_{T} c_{r}}}{\alpha} \int_{\frac{E_{c}}{k T}}^{\infty} f\left(\frac{E_{c}}{k T}\right) P_{r}\left(\frac{E_{c}}{k T}\right) d\left(\frac{E_{c}}{k T}\right)=\frac{\overline{\sigma_{T} c_{r}}}{\alpha} \overline{P_{r}}
$$

For the theoretical data, the rate constant is evaluated by Eq. (4) and the reaction probability is calculated by the following equation:

$$
\overline{P_{r}}=\frac{k_{f} \alpha}{\overline{\sigma_{T} c_{r}}}, \quad \alpha=1 \text { for } A \neq B, \quad \alpha=2 \text { for } A=B
$$

\section{B. Recombination reaction}

In the DSMC method, collisions are usually considered as binary. It represents a challenge to simulate threebody recombination reactions. In Vincenti and Kruger [17], it is stated that, for the recombination reaction between two nitrogen atoms and any third body $\mathrm{M}$,

The rate of formation of $\mathrm{N}_{2}$ is governed by

$$
N+N+M \rightarrow N_{2}+M
$$

$$
\frac{d n_{N_{2}}}{d t}=\left(k_{b} n_{N} n_{N}\right) n_{M}
$$

$n_{N}$ and $n_{M}$ are the number densities of nitrogen atom and the third body $\mathrm{M}$, respectively. $k_{b}$ is the backward rate coefficient, which can be written as $k_{b}=k_{f} / K_{c}=\left(a_{f} / a_{c}\right) T^{b_{f}-b_{c}} \cdot a_{f}$ and $b_{f}$ are constants for forward rate reaction, and $a_{c}$ and $b_{c}$ are constants for equilibrium rate reaction. The parentheses in Eq. (11) indicated that, in the new recombination model for the DSMC method, the reaction is first treated as a binary collision between two $\mathrm{N}$ atoms. This allows the derivation of a recombination probability in the same way as for a dissociating reaction in the previous section. The correct reaction probability must then be multiplied by the number density of the third body 
$n_{M}$. The third body is chosen randomly from those that exist in the cell. It can be any species in the cell. Thus, the number density of the third body is the total number density. The derivation of the theoretical reaction probability and rate constants are described in the following [9]:

$$
\begin{aligned}
& \frac{d n_{N_{2}}}{d t}=\left(k_{b} n_{N} n_{N}\right) n_{M}=\left(\overline{P_{r, b}} \cdot Z_{N, N}\right) \cdot n_{M}=\overline{P_{r, \text { new }}} \cdot Z_{N, N}, \\
& \text { that is, } \overline{P_{r, \text { new }}}=\overline{P_{r, b}} \cdot n_{M}
\end{aligned}
$$

$\overline{P_{r, b}}$ is the probability of binary collision between two atoms. $\overline{P_{n e w, b}}$ is the new probability of the recombination reaction. $Z_{N, N}$ is the collision rate between nitrogen atoms. From Eq. (12), the rate constant can be obtained as follows:

$$
\left(k_{b} n_{N} n_{N}\right) n_{M}=\overline{P_{r, \text { new }}} \cdot Z_{N, N}=\overline{P_{r, \text { new }}} \cdot \frac{n_{N} \cdot n_{N} \cdot \overline{\sigma g}}{\alpha}
$$

For the DSMC method, the form of the binary recombination probability is chosen as [9]

$$
\begin{aligned}
& P_{r, b}=\left(\frac{1}{Z_{r}}\right) E_{c}^{\chi}, \\
& \frac{1}{Z_{r}}=\frac{\sqrt{\pi}}{2^{1 / 2-\gamma}} \cdot \frac{a_{f} / a_{c}}{k^{b_{f}-b_{c}}} \cdot \frac{m_{r}^{1 / 2-\gamma}}{\sigma_{r e f}} \cdot\left(\frac{m_{r}}{2(2-\omega) k T_{r e f}}\right)^{\gamma} \cdot \frac{\Gamma\left(7 / 2+\zeta_{M} / 2-\gamma\right)}{\Gamma\left(7 / 2+\zeta_{M} / 2-\gamma+\chi\right)}
\end{aligned}
$$

which $\chi=b_{f}-c_{f}-1 / 2+\gamma . E_{c}$ is the total energy of these three particles. $\zeta_{M}$ is the degree of freedom in internal mode for the third-body particle. The new recombination probability will be obtained by multiplying $n_{M}$, that is, $P_{n e w, b}=P_{r, b} \cdot n_{M}$.

For the DSMC simulation, the mean recombination probability is obtained in the same way as the dissociation method. Then the rate constant can be determined from Eq. (13), that is,

$$
k_{b}=\frac{\overline{P_{r, \text { new }}} \cdot \overline{\sigma g}}{\alpha \cdot n_{M}}, \quad \alpha=2 \text { for } A=B
$$

For theoretical results, the rate constant is determined from the Arrhenius equation and the reaction probability can be calculated by the following equation

$$
k_{b}=a T^{b} \exp \left(-\frac{\theta}{T}\right), \quad P_{n e w, b}=\frac{\alpha \cdot k_{b} \cdot n_{M}}{\overline{\sigma g}}, \quad \alpha=2 \text { for } A=B
$$

\section{Exchange reaction}

There are several reactions that exchange the atom in air under high temperature conditions. If a molecule $\mathrm{AB}$ reacts with an atom $\mathrm{C}$ to form a molecule $\mathrm{AC}$ and an atom $\mathrm{B}$, which is an exchange reaction, which formula reaction is

$$
A B+C \leftrightarrow A C+B
$$

The derivations of the probability of exchange reaction are the same as the dissociation reaction, which as mentioned above. Thus, we do not repeat here. The only difference of using DSMC between dissociation and exchange reactions is in the setting of the chem.dat file, which is the information of reaction constants.

No matter what kind of chemical reaction is, the Larsen-Borgnakke model [18] is applied to redistribute the translational and internal energies;

\section{Verification}

A single two-dimensional cell is considered as the first benchmark to calculate the probability of each chemical reaction, in which only one reaction (direction) is permitted. All the walls are diffusive and the temperature is the same as the initial temperature. The range of temperature is from 3,000 to $13,000 \mathrm{~K}$. The value of the initial number density is assigned as $\rho_{\mathrm{d}} / \rho=10^{5}$. $\rho_{\mathrm{d}}$ is the characteristic density for dissociation, which is a function of temperature [17]. Figure 2 shows the simulated probabilities of some typical dissociation, exchange and recombination reactions 
with different temperatures. To calculate the reaction probability (Pr), the numbers of collisions between reactants, $\mathrm{A}$, and collisions that do react, $\mathrm{B}$, are accumulated through the DSMC simulation. The reaction probability can be easily calculated as the ratio of $\mathrm{B}$ to $\mathrm{A}(\mathrm{B} / \mathrm{A})$. The simulated results generally agree very well with the theoretical data in Fig. 2. For dissociation reaction, the probability is increased with increasing temperatures because the flow has enough energy to activate the chemical reactions. But the recombination has the opposite situation because atoms are too active to form chemical bond when temperature increased.

The second benchmark is to simulate pure species $\left(\mathrm{N}_{2}\right.$ or $\left.\mathrm{O}_{2}\right)$ with forward and backward reactions under different number densities and temperatures, which are shown in Fig. 3. The degree of dissociation is defined as Eq. (18);

$$
\alpha \equiv \frac{N^{a}}{N_{A}}=\frac{m N^{a}}{m N_{A}}=\frac{\text { mass of dissociated } A-\text { atoms }}{\text { total mass of gas }}
$$

$N^{a}$ and $N_{A}$ are the particle number of dissociated A-atoms and the total number of A-atoms present in the mixture, respectively. The ranges of temperature of nitrogen and oxygen gas are 5,000 13,000 and 3,000 7,000 K, respectively. Three different initial number densities are used for these simulations, which are $\rho_{\mathrm{d}} / \rho=10^{5}, 10^{6}$, and $10^{7}$. As can be seen, the degree of dissociation increases with increasing temperatures and rarefied gas. The agreement with theoretical values [17] are remarkably well. From the results of the above benchmark tests, it shows that current chemical-reaction module is correctly implemented in the PDSC.

\section{Applications}

In this section, three different objects in geometry are simulated by the current PDSC. All of these applications are simulated with 34 chemical reactions [19], including 14 dissociations, 14 recombinations and 10 exchanges. Adaptive mesh refinement, the variable time step scheme and dynamic domain decomposition are applied in these simulations to obtain more accurate results and reduce the enormous computational time. The rotational energy is redistributed by the Larsen-Borgnakke model with relaxation number of 5. But the redistribution of vibrational energy is based on the quantum vibrational model and the vibrational relaxation number is set as 50, which is different from the references. Related information about quantum vibrational model can be referred to Bird's book [2]. Each application is described in the following separately;

\section{A. Sphere flow}

The first application is a hypersonic re-entry sphere flow, which was simulated by Dogra et al.. Related flow conditions are listed as follows [6]. The altitude of this case is $90 \mathrm{~km}$ and the free-stream density is $3.43 \mathrm{E}-6 \mathrm{~kg} / \mathrm{m}^{3}$; the initial mole fractions of oxygen molecule, nitrogen molecule and oxygen atoms are 0.209, 0.788 and 0.004, respectively; the free-stream velocity and temperature are $7,500 \mathrm{~m} / \mathrm{s}$ and $188 \mathrm{~K}$, respectively; the wall temperature is $350 \mathrm{~K}$ with fully diffuse surfaces; the corresponding Knudsen number $K n$ and Reynolds number $R e$ (based on the diameter of the sphere, which is 1.6 meter) are 0.01 and 3,243, respectively. One sixteenth of the whole domain is used in this simulation to save the computational time because it is an axis-symmetric flow. A two-level adaptive mesh, which is shown in Fig. 4, is used and the cell number and particle number are about 670,000 and 3,000,000, respectively.

Figure 5a $5 \mathrm{c}$ illustrate flow field contours of this simulation, which including normalized density, normalized overall temperature and Mach number on the X-Z plane, respectively. The normalized properties are based on the free-stream condition. As we can see, there is a very strong bow shock standing in the front of the sphere and the wake region exists behind the object. The normalized density and overall temperature are increased along the $\mathrm{X}$-axis first because the bow shock effect, which the maximum value are about 120 and 60, respectively. Figure 6 shows the surface coefficients, including pressure, skin-friction and heat transfer coefficients, along the object surface. This figure is plotted as a function of the circumferential angle $(\theta)$ measured clockwise from the stagnation point and the simulated results of Dogra [6] are also plotted for comparisons. The pressure (Fig. 6a) and heat transfer (Fig. 6c) coefficients decreased with increasing circumferential angle and the maximum value are at stagnation point $\theta=0^{\circ}$. The simulated result agrees very well with the reference [6]. The skin-friction coefficient (Fig. 6b) increased then decreased along the sphere surface and the maximum value is near $\theta=40^{\circ}$. As we can see the results of skin-friction and heat transfer coefficients are pretty scattering because the particle sampling is not enough. Although the results are not exactly the same, the results are acceptable by comparing with reference. Figure 7 a demonstrates the mole fractions of each species, including $\mathrm{N}_{2}, \mathrm{O}_{2}, \mathrm{NO}, \mathrm{N}$ and $\mathrm{O}$, along the stagnation line. From this figure we can clearly understand there is no chemical reaction near the free-stream region because the temperature is too low to have 
chemical reactions. Then the amounts of NO, N and $\mathrm{O}$ are increased when the flow approaches the bow shock region due to the chemical reactions, which mostly are from dissociation and exchange reactions. Figure $7 \mathrm{~b}$ is the distribution of translational, rotational and vibrational temperatures, which shows there is significant nonequilibrium phenomenon near the shock region. The current results have lower vibrational temperature and higher translational and rotational temperatures before $\mathrm{x} \sim-0.04$ because different energy redistribution model is used. Physically, the temperature and collision events should not be enough to transfer energy from the translational mode to the vibrational mode. That is why the present result in Fig. 7a has higher mole fractions in nitrogen and oxygen molecules than Dogras's results. Although both of these figures do not exactly agree with the reference, the current results have the same trend that reasonable and acceptable.

\section{B. Blunt-body flow}

The second application is a hypersonic flow over a blunt-body with zero angle of attack, which is simulated by Dogra et al. in 1995 [20]. Related flow conditions are listed as follows; The altitude of this case is $85 \mathrm{~km}$ and the free-stream density is $7.955 \mathrm{E}-6 \mathrm{~kg} / \mathrm{m}^{3}$; the initial mole fractions of oxygen molecule and nitrogen molecule are 0.2372 and 0.7628 , respectively; the free-stream velocity and temperature are $7,000 \mathrm{~m} / \mathrm{s}$ and $180.65 \mathrm{~K}$, respectively; the wall temperature is $1000 \mathrm{~K}$ with fully diffuse surfaces; the corresponding Knudsen number $\mathrm{Kn}$ and Reynolds number $\operatorname{Re}$ (based on the maximum diameter of the vehicle, which is 2 meter) are 0.00359 and 9,033, respectively. Evolution of adaptive mesh with cell quality control is presented in Fig. 8. We can see there is a very clear bow shock in the front of the blunt body. The two-level cell number and particle number are about 730,000 and $20,000,000$ respectively.

Figure 9 illustrates normalized flow field contours of this simulation based on the free-stream condition. The flow-field is similar to the sphere flow above that we do not repeat here. We also can see there exists a very strong bow shock in the front of the sphere and a wake region exists behind the blunt-body. The normalized density and overall temperature are increased along the $\mathrm{X}$-axis first because the bow shock effect, which the maximum values are about 220 and 65, respectively. Figure 10 shows the contours of mole fraction for each species. The initial species, $\mathrm{N}_{2}$ and $\mathrm{O}_{2}$, are dissociated and exchanged by collisions with high temperature. $\mathrm{NO}, \mathrm{N}$ and $\mathrm{O}$ are created as shown in Fig. 10c-10e. Note that the mole fraction of oxygen atom is higher than that of nitrogen atom because the dissociation temperature and activation energy of oxygen molecule are lower. The mole fraction of NO is least because it can only be created by exchange and recombination reactions, which monoatomic species have fewer relative particles. Although the distribution of these three species are quiet similar, it is very interesting that the maximum values of products are flowed down to the downstream and located at different location.

\section{Apollo command module}

The last application is a realistic three-dimensional Apollo command module case. Only half of the whole Apollo spacecraft is simulated by taking advantage of the geometrical symmetry to save the computational cost. Related flow conditions are summarized as follows. The altitude is $100 \mathrm{~km}$, while the angle of attack is $25^{\circ}$. The number densities of free-stream gases, including nitrogen, oxygen and oxygen atom, are 8.467E18, 2.025E18 and 3.995E17, respectively. Note that these number densities are determined by the MSIS-E-90 Atmosphere model (http://nssdc.gsfc.nasa.gov/space/model/models/msis.html). In addition, the velocity and temperature are 9,592.64 $\mathrm{m} / \mathrm{s}$ and $190.6 \mathrm{~K}$, respectively. Wall temperature is fixed and is predicted by the Stephan-Boltzmann Law, which is 1,378 K. Resulting Knudsen number $K n$ and Reynolds number $R e$ (based on the radius of aft compartment) are about 0.034 and 1528, respectively. Cell numbers and particle numbers of this simulation are approximately 780,000 and $11,000,000$, respectively. PDSC is run on a 10-node PC-cluster system using Linux OS and MPI at our laboratory.

Surface mesh distribution is shown in Fig. 11a. Figure 11b is the instantaneous distribution of particles near the symmetric plane. Highly packed particles are found around the front face of the Apollo spacecraft due to the abrupt increase of density in the stagnation regime. In contrast, much fewer particles appear behind the spacecraft that causes the very low pressure in the wake region. Figure 12 also shows the normalized contours distribution, including density, overall temperature and Mach number, based on the free-stream flow conditions. The maximum normalized values for these three properties are about 120, 65 and 34, respectively. Figures 13a-13e represent the distributions of mole fractions of $\mathrm{N}_{2}, \mathrm{O}_{2}, \mathrm{NO}, \mathrm{N}$ and $\mathrm{O}$, respectively, on the symmetrical plane of the Apollo case. In these figures, a strong bow shock is also clearly shown in front of the spacecraft, while a highly rarefied region is formed behind the spacecraft. NO and $\mathrm{N}$ species are also created due to chemical reactions in the front of the spacecraft because of the strong bow shock. In these figure, we see that the nitrogen atoms and oxygen atoms are also convected downstream as in the previous blunt-body case. 


\section{Conclusions}

Chemical reactions are important for the rarefied gas dynamics when the flow speed and temperature are very high, which is common in launching, re-entry and missile flight conditions. Some material processing also has chemical reactions that can not be negligible. To simulate these applications, the direct simulation Monte Carlo is an alternative method which can couple chemical-reaction function easily in the molecular level. In the current study, a general-purpose parallel DSMC code (PDSC) with chemistry is proposed and validated by comparing reaction probabilities and degree of dissociation of a 2-D single cell. It can be seem the simulated results agree with the theoretical solutionsvery well. Then, three 3-D challenging hypersonic re-entry flows, which are the spherical-body, blunt-body and Apollo Command Module, are simulated and discussed. For these cases, a strong bow shock and a wake region are stranded before and behind the objects, respectively. Also, the surface aerodynamic coefficients of the re-entry sphere and mole fraction of each species along the stagnation line are calculated and compared with those of Dogra. The simulated results are remarkable although the skin-friction and heat transfer coefficients do not exactly agree because insufficient samplings and different energy transfer model were used. All of these verification and demonstrations show the chemical-reaction module has been well-developed in the current PDSC.

\section{Acknowledgments}

I would like to thank Professor I. D. Boyd at the University of Michigan in the United States for assisting to study chemical-reaction function of DSMC. I am also grateful for the discussions with Professor Wu at the National Chiao-Thung in Taiwan. Finally, I would like to appreciate the support of the National Space Organization (NSPO) in Taiwan.

\section{References}

${ }^{1}$ Bird G. A., "Molecular Gas Dynamic”, Clarendon Press, Oxford, UK, 1976.

${ }^{2}$ Bird G. A., Molecular Gas Dynamics and the Direct Simulation of Gas Flows, 1st edition, Clarendon Press, Oxford, New York, 1994.

${ }^{3}$ Lee Y. K., and Lee J. W., "Direct simulation of compression characteristics for a simple drag pump model," Vacuum, Vol. 47, 1996, pp. 807-809.

${ }^{4}$ Alexander F. J., Garcia A. L., and Alder B. J., "Direct simulation Monte Carlo for thin-film bearings," Physics of Fluids A, Vol. 6, 1994, pp. 3854-3860.

${ }^{5} \mathrm{Wu}$ J.-S., and Tseng K.-C., "Analysis of micro-scale gas flows with pressure boundaries using direct simulation Monte Carlo method," Computers \& Fluids, Vol. 30, 2001, pp. 771-725.

${ }^{6}$ Dogra V. K., Wilmoth R. G., and Moss J. N., “Aerothermodynamics of a 1.6-Meter-Diameter Sphere in Hypersonic Rarefied Flow,” AIAA Journal, Vol. 30, No. 7, 1992, pp. 1789-1794.

${ }^{7}$ Bartel T. J., and Johannes J. E., "Trace chemistry modeling with DSMC in chemically reacting plasmas," AIAA-1998-2753, 1998.

${ }^{8}$ Kruis F. E., Maisels A., and Fissan H., "Particle Technology and Fluidization Direct simulation Monte Carlo method for particle coagulation and aggregation," AIChE Journal, Vol. 46, Issue 9, 2004, pp. 1735-1742.

${ }^{9}$ Boyd I. D., "Analysis of vibration-dissociation-recombination processes behind strong shock waves of nitrogen," Physics of Fluids A, Vol. 4, No. 1, 1992, pp. 178-185.

${ }^{10}$ Gimelshein S. F., Gimelshein N. E., Ivanov M. S., and Wysong I.J., "On the use of chemical reaction rates with discrete internal energies in the direct simulation Monte Carlo method," Physics of Fluids, Vol. 16, No. 7, 2004, pp. $2442-2451$.

${ }^{11}$ Nanbu K., "Theoretical Basis on the Direct Monte Carlo Method," Rarefied Gas Dynamics, Vol. 1, Teubner, Stuttgart, 1986.

${ }^{12}$ Wagner W., "A convergence proof for Bird's direct simulation Monte Carlo method for the Boltzmann equation," Journal of Statistic Physics, Vol. 66, No. 3/4, 1992, pp. 1011-1044.

${ }^{13}$ Wu J.-S., Tseng K.-C., Lee U.-M., and Lian Y.-Y., "Development of a General Parallel Three-Dimensional Direct Simulation Monte Carlo Code (PDSC)”, 24th International Symposium on Rarefied Gas Dynamics, Bari, Italy, July 10-16, 2004.

${ }^{14}$ Wu J.-S., Tseng K.-C., and Wu F.-Y., "Parallel Three-Dimensional DSMC Method Using Mesh Refinement and Variable Time-Step Scheme," Computer Physics Communications, Vol. 162, No. 3, 2004, pp. 166-187.

${ }^{15} \mathrm{Wu}$ J.-S., and Tseng K.-C., "Parallel DSMC Method Using Dynamic Domain Decomposition," International Journal for Numerical Methods in Engineering, Vol. 63, 2005, pp. 37-76.

${ }^{16}$ Boyd I. D., and Stark J. P. W., "Direct Simulation of Chemical Reactions," Journal of Thermophysiscs and Heat Transfer, Vol. 4, No. 3, 1990, pp. 391-393.

${ }^{17}$ Vincenti W. G., and Kruger C. H., Introduction to Physical Gas Dynamics, Wiley, New York, 1965.

${ }^{18}$ Borgnakke, C., and Larsen P. S., "Statistical collision model for Monte Carlo simulation of polyatomic gas mixture," Journal of Computational Physics, Vol. 18, 1975, pp. 405-420. 
${ }^{19}$ Dorothy B. L., and Winston D. G., "The aerothermodynamic environment of the Apollo command module during superorbital entry," NASA TND-6792, 1972.

${ }^{20}$ Dogra V. K., Moss J. N., Wilmoth R. G., Taylor J. C., and Hassan H. A., "Effects of Chemistry on Blunt-Body Wake Structure," AIAA Journal, Vol. 33, No. 3, 1995, pp. 463-469.

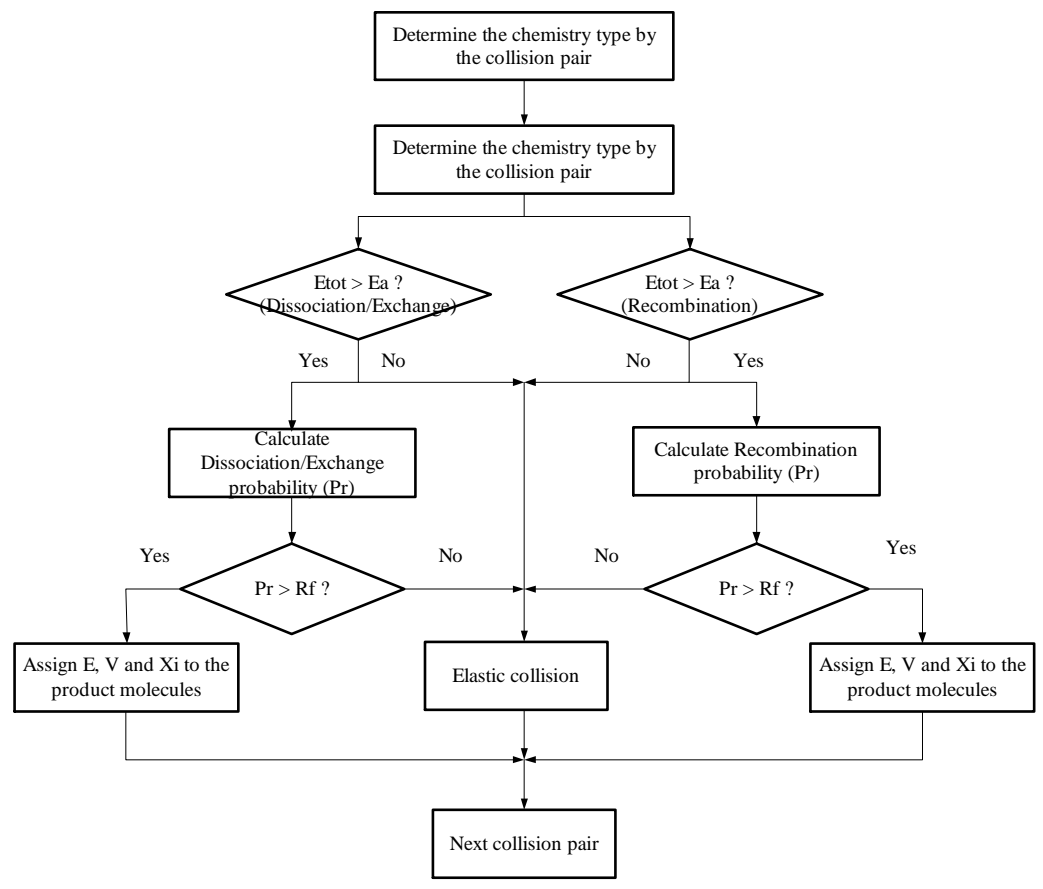

Figure 1. The flow chart of chemical reaction in the PDSC.

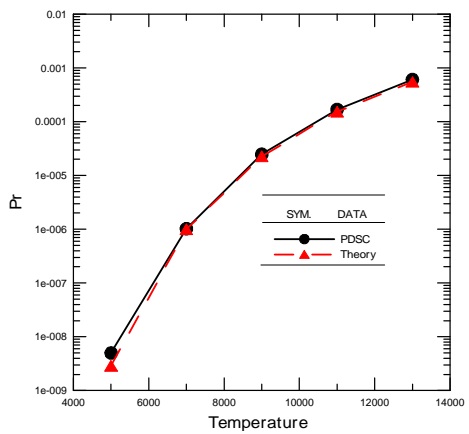

(a) $\mathrm{N}_{2}+\mathrm{N}_{2}->\mathrm{N}+\mathrm{N}+\mathrm{N}_{2}$

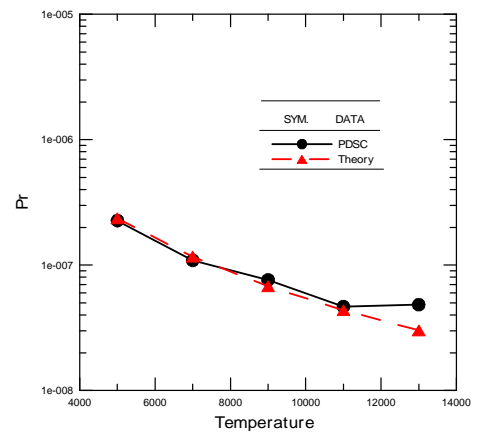

(b) $\mathrm{N}+\mathrm{N}+\mathrm{N}_{2}->\mathrm{N}_{2}+\mathrm{N}_{2}$

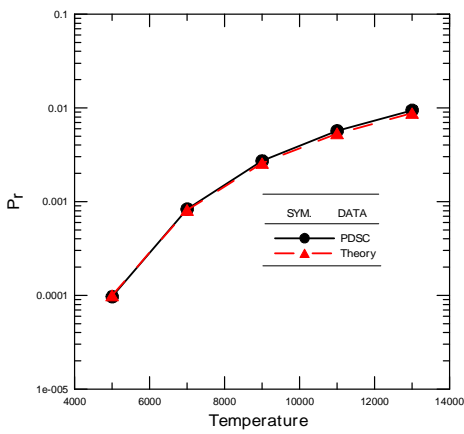

(c) $\mathrm{N}_{2}+\mathrm{O}->\mathrm{NO}+\mathrm{N}$

Figure 2. Probabilities for typical chemical reactions (a) dissociation; (b) recombination; (c) exchange.

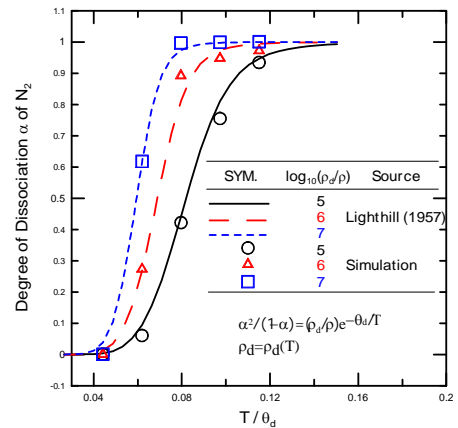

(a)

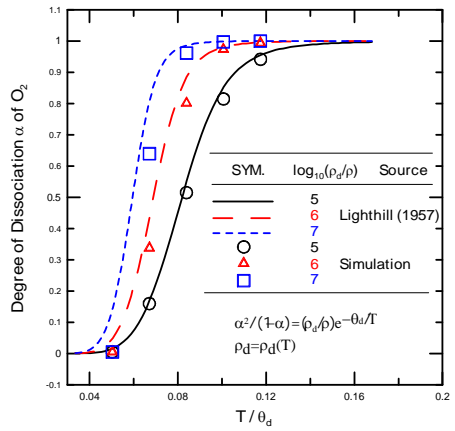

(b)

Figure 3. Degree of dissociation for idea dissociating gas (a) nitrogen; (b) oxygen. 


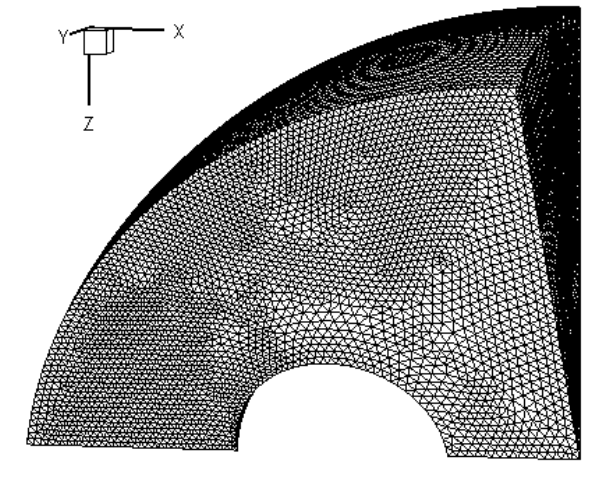

(a)

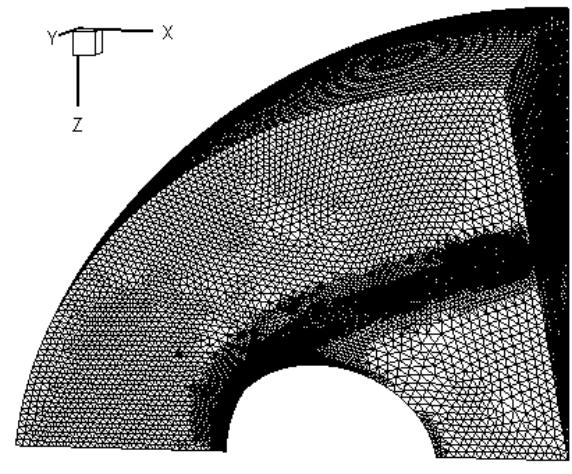

(b)

Figure 4. Evolution of the adaptive mesh for the re-entry sphere flow (a) initial $(100,476)$; (b) level-2 $(669,072)$.

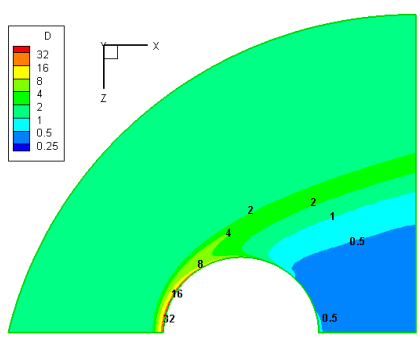

(a)

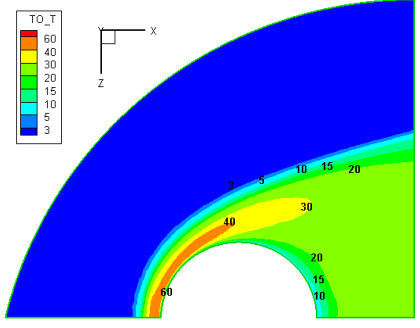

(b)

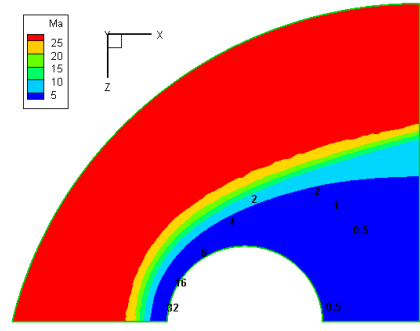

(c)

Figure 5. Normalized property contours of the re-entry sphere flow (a) density; (b) overall temperature; (c) Mach number.

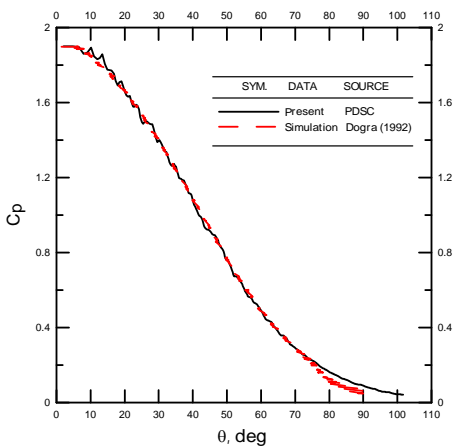

(a)

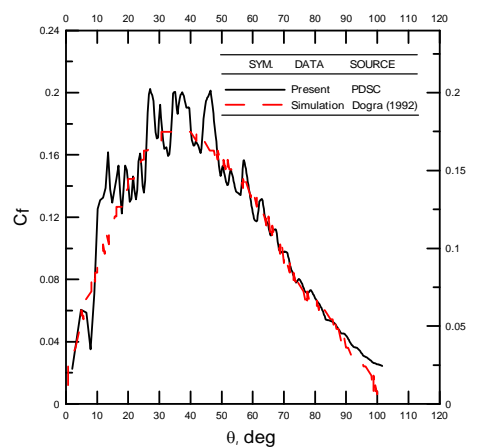

(b)

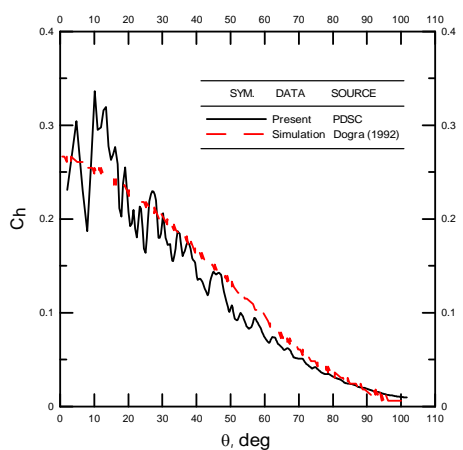

(c)

Figure 6. Surface coefficients of the re-entry sphere (a) pressure; (b) skin-friction; (c) heat transfer. 


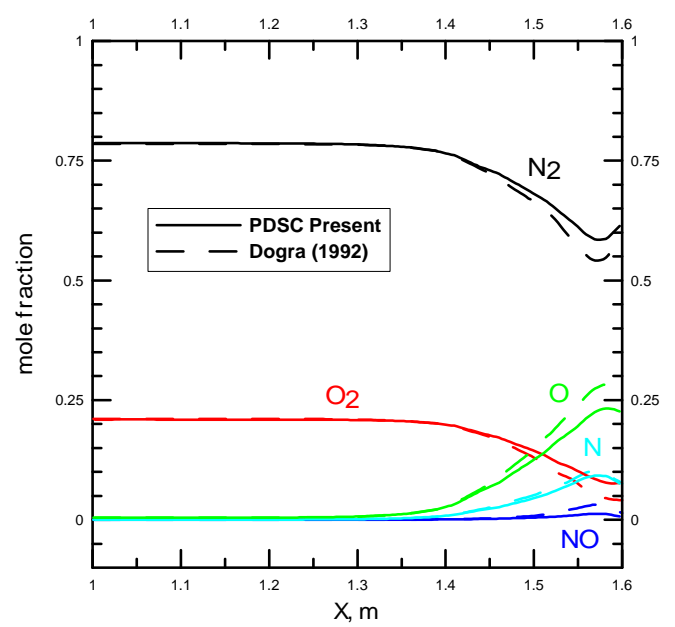

(a)

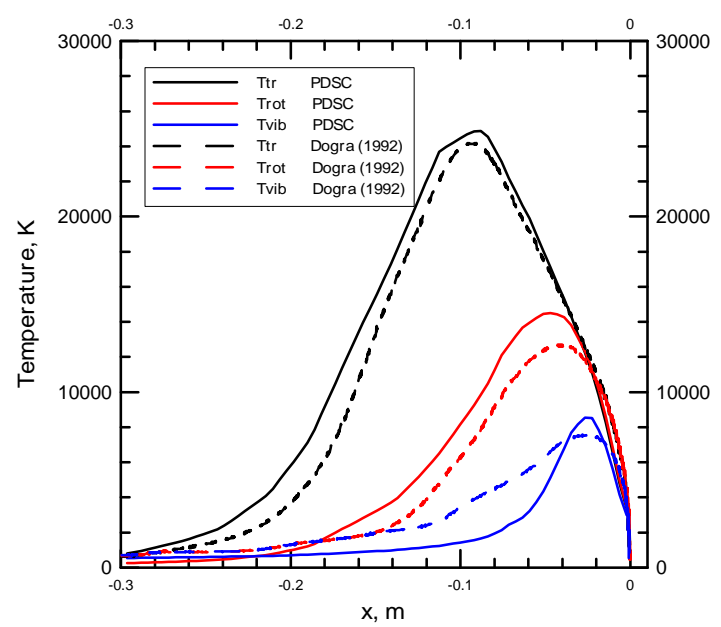

(b)

Figure 7. Flow properties along the stagnation line for the re-entry sphere flow (a) mole fraction; (b) temperatures.

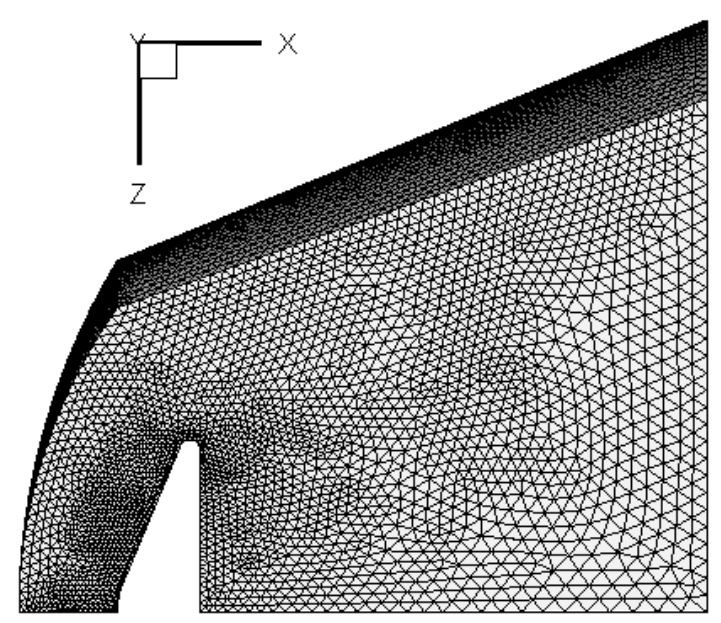

(a)

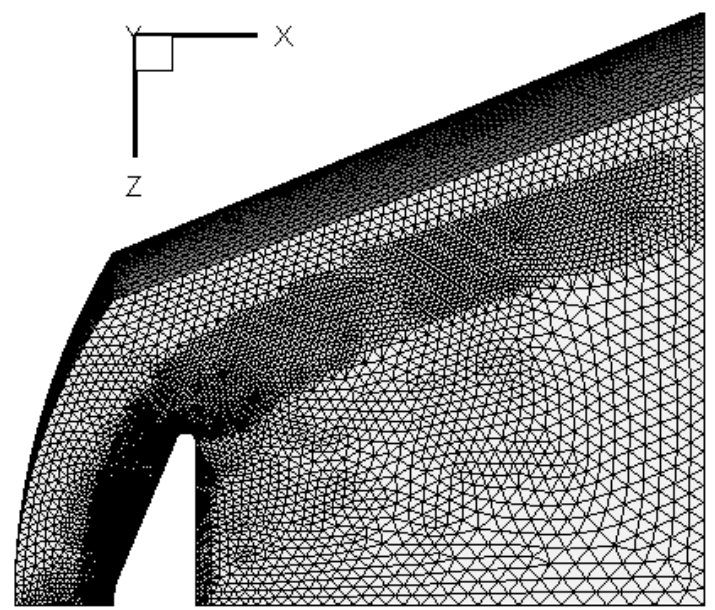

(b)

Figure 8. Evolution of the adaptive mesh for the re-entry blunt-body flow (a) initial $(\mathbf{7 4 , 8 3 0 )}$; (b) level-2 $(\mathbf{7 2 5 , 6 6 7 ) .}$

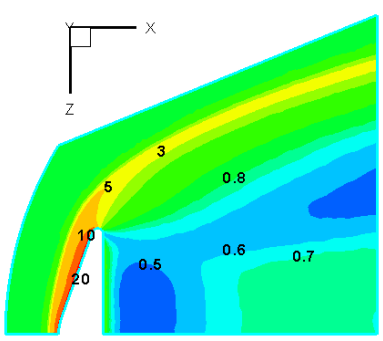

(a)

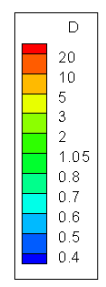

Figure 9. Normali

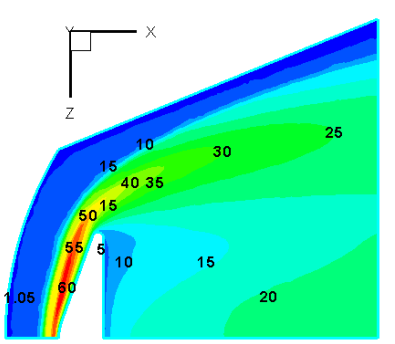

(b)

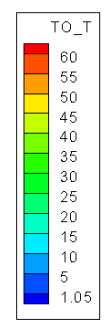

(a)

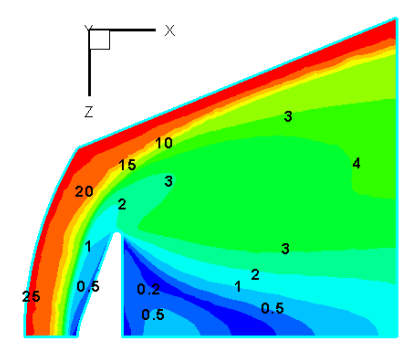

(c) (c) Mach number. 


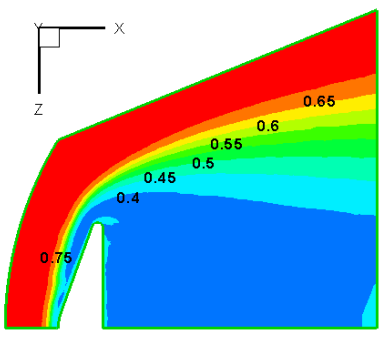

(a)

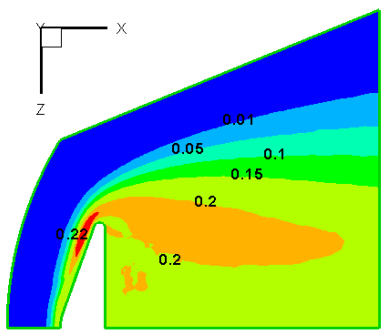

(d)

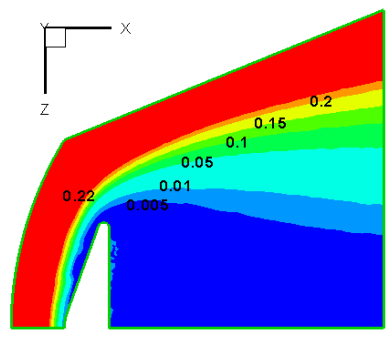

(b)

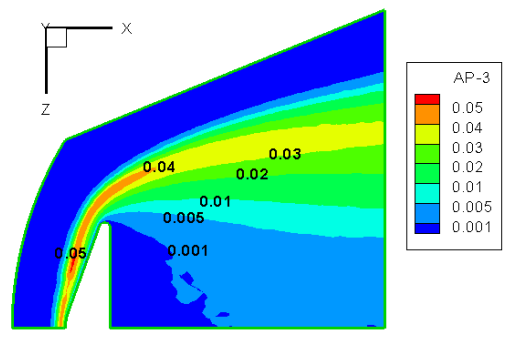

(c)
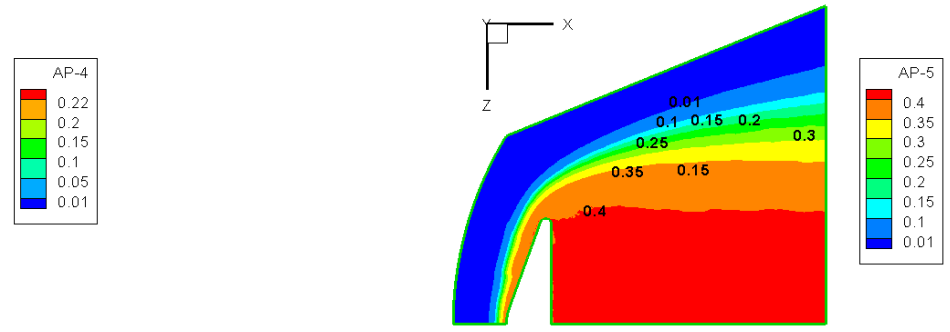

(e)

Figure 10. Mole fraction contours of the re-entry blunt-body flow (a) $\mathrm{N}_{2} ;$ (b) $\mathrm{O}_{2}$; (c) $\mathrm{NO}$; (d) $\mathrm{N}$; (e) $\mathrm{O}$.

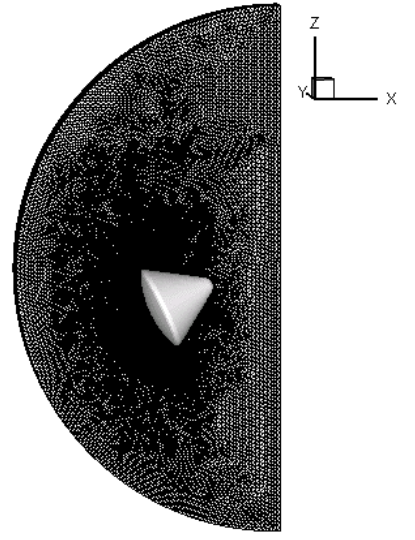

(a)

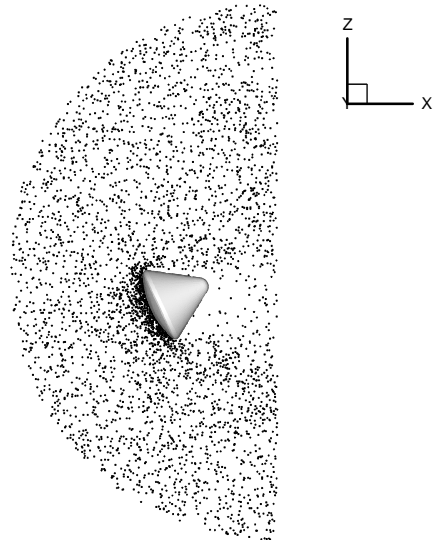

(b)

Figure 11. Mesh and particle distribution of the 3-D Apollo case on X-Z plane (a) mesh; (b) particle distribution.

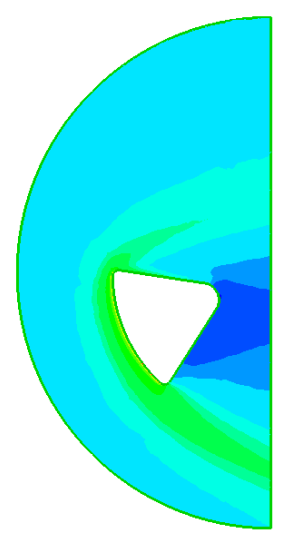

(a)

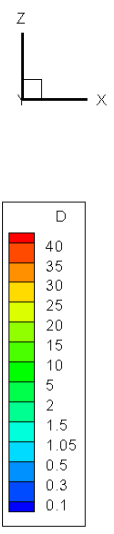

(b)

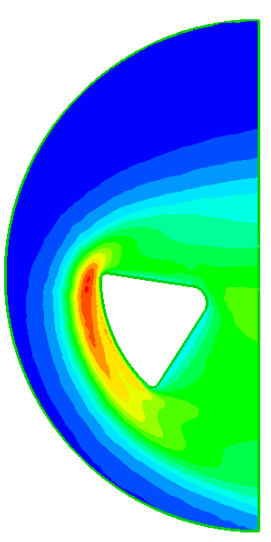

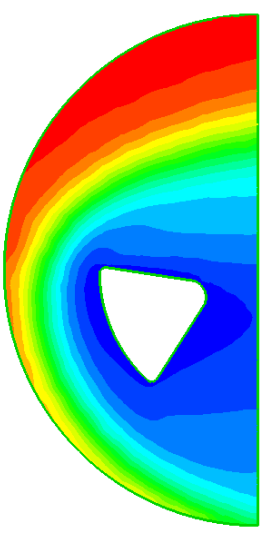

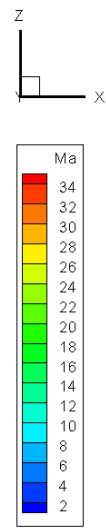

(c)

Figure 12. Normalized property contours of the re-entry blunt-body flow (a) density; (b) overall temperature; (c) Mach number. 


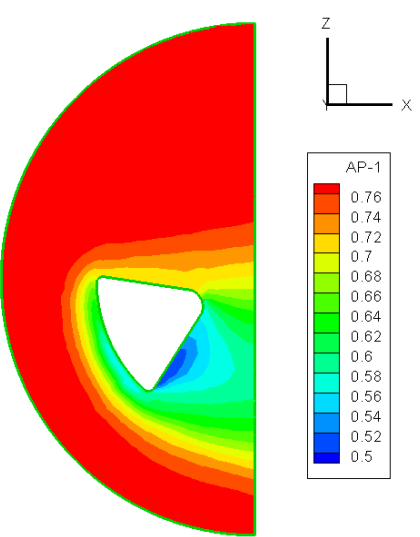

(a)

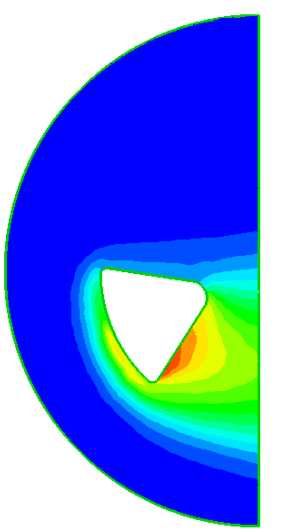

(d)

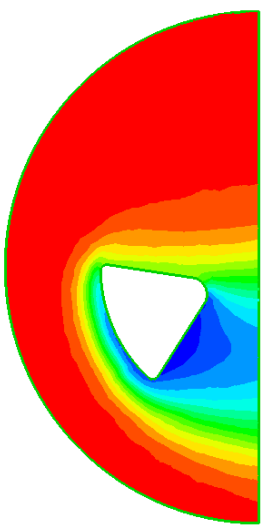

(b)

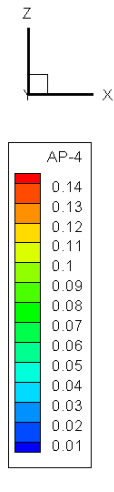

Figure 13. Mole fraction contours of the Apollo case (a) $\mathrm{N}_{2} ;$ (b) $\mathrm{O}_{2} ;$ (c) $\mathrm{NO}$; (d) $\mathrm{N}$; (e) $\mathrm{O}$.

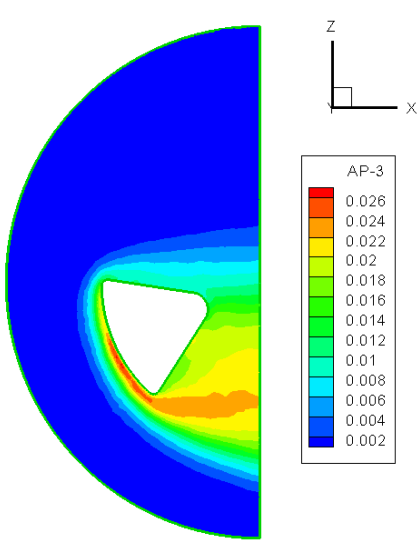

(c)

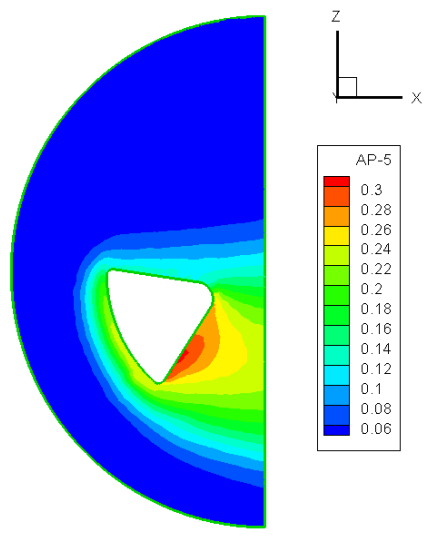

(e) 